\title{
Teleportation of an atomic momentum state
}

\author{
Shahid Qamar, ${ }^{1,2}$ Shi-Yao Zhu, ${ }^{3}$ and M. Suhail Zubairy ${ }^{1,4}$ \\ ${ }^{1}$ Institute for Quantum Studies and Department of Physics, Texas A\&M University, College Station, Texas 77843-4242 \\ ${ }^{2}$ Department of Physics and Applied Mathematics, Pakistan Institute of Engineering and Applied Sciences, \\ Nilore, Islamabad, Pakistan \\ ${ }^{3}$ Department of Physics, Hong Kong Baptist University, Hong Kong, China \\ ${ }^{4}$ Department of Electronics, Quaid-i-Azam University, Islamabad, Pakistan
}

(Received 12 December 2002; published 22 April 2003)

\begin{abstract}
In this paper, we propose a scheme for teleportating a superposition of atomic center-of-mass momentum states to a superposition of the cavity field using quantum controlled-NOT gate via atomic scattering in the Bragg regime and cavity quantum electrodynamics.
\end{abstract}

DOI: 10.1103/PhysRevA.67.042318

PACS number(s): 03.67.-a, 42.50.Ct, 32.80.Qk

Teleportation of an unknown quantum state which is based upon the idea of quantum nonlocality [1,2] lies at the center of quantum-information theory. Since the pioneering contribution of Bennett et al. [3], a number of proposals have been presented for the teleportation of both atomic and field states, based on cavity QED $[4,5]$. In some recent work, teleportation of an entangled multiparticle state and coherent superposition of $2^{n}$ fock states have also been presented $[6,7]$. In addition to the schemes for discrete variables, the idea was extended for teleportation of continuous variables $[8-10]$. Following theoretical progress in the field of quantum teleportation, its experimental verification has also been realized in some recent experiments. For example, teleportation by producing pairs of entangled photons using parametric down-conversion [11,12], teleportation of an optical coherent state using squeezed state entanglement [13], and a vacuum-one-photon qubit represented by a mode of the electromagnetic field [14].

In most of the recent studies, teleportation has been considered for photon polarization [3,8,11,12], optical field quadratures [9,13], and multiatom spin components [15]. However, an interesting question is how to teleport the information related to the center-of-mass wave function of a quantum state. In some recent studies, this question has been addressed for continuous quantum variables. For example, Opatrny and Kurizki have proposed an interesting scheme for the teleportation of the translational center-of-mass quantum state (matter-wave packet) by molecular dissociation and collision [16]. In another study, Parkins and Kimble have presented a scheme for teleporting the center-of-mass wave function of trapped atoms [17]. Their scheme is based upon the coupling between the motion of an atom trapped inside a cavity and external propagating light field. In this paper, we consider a scheme for teleporting an unknown superposition of discrete momentum states corresponding to the atomic center-of-mass motion to a superposition of the cavity field. The measurement of Bell states are realized by using atomic scattering through standing field in the Bragg regime and then applying cavity QED.

The momentum state that is to be transferred is defined as

$$
\left|\Psi_{a}\right\rangle=c_{a}\left|+p_{0}\right\rangle+c_{b}\left|-p_{0}\right\rangle
$$

where $\left|+p_{0}\right\rangle$ and $\left|-p_{0}\right\rangle$ are the momentum states of the incoming atoms in $\pm x$ directions, respectively (see Fig. 1) and $c_{a}$ and $c_{b}$ are the corresponding probability amplitudes. The initial superposition of momentum eigenstate defined by Eq. (1) can be realized by considering an atom with a large position spread that passes through a standing wave in the Bragg regime. The superposition may be known or unknown and the probability amplitudes will depend upon the passage time through the standing wave. There may be errors as the atom cannot be in a pure momentum state (with infinite spread) and our analysis may be a good approximation only if the spread is larger than the wavelength of the standingwave field.

Our proposed scheme is as follows. First, we prepare two cavities in the entangled Fock states $|0\rangle$ and $|1\rangle$. This can be achieved by sending across both the cavities (initially in a vacuum state) a two-level atom resonant with the cavity field and prepared in an excited state [4]. The atom is made to undergo a $\pi / 2$ pulse in the first cavity and a $\pi$ pulse in the second cavity. This can be achieved by properly choosing the interaction times of the atom with the field in the two cavi-

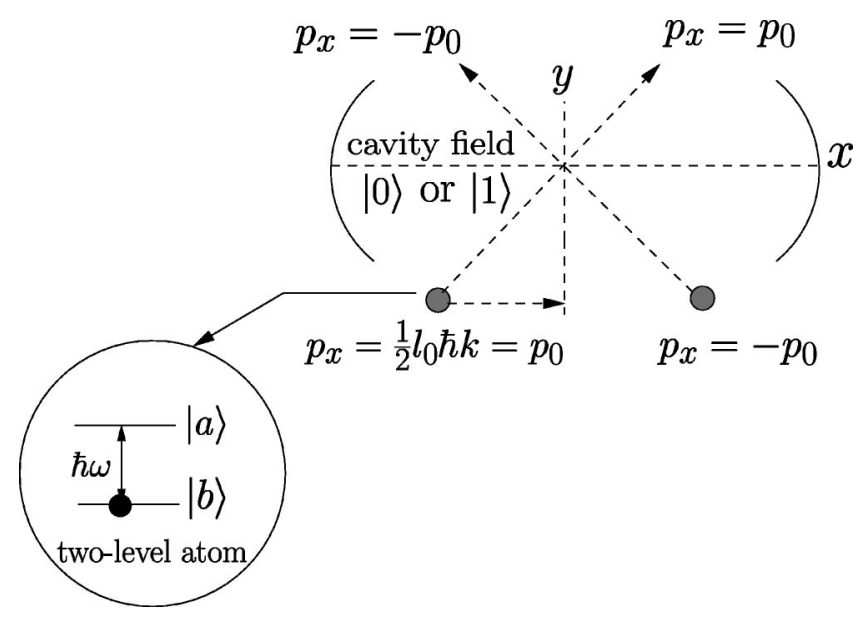

FIG. 1. Schematic diagram of the quantum controlled-NOT logic gate used for the atomic momentum state teleportation. If $p_{0}$ is the moment of the incoming atom along the $x$ axis, then it changes into $-p_{0}$ if there is a one-photon state inside the cavity, and remains $p_{0}$ if the cavity field is in a vacuum state. 
ties. When the atom undergoes a $\pi / 2$ pulse in the first cavity, then it will be in a superposition of excited and ground states correlated with the cavity in zero- and one-photon states. The second cavity remains in a vacuum state. If the atom is still in the excited level after leaving the first cavity in the vacuum state, then it will undergo a transition from an excited to a ground state by a $\pi$ pulse with unit probability and exit the second cavity after leaving one photon. If it contributes a photon in the first cavity and leaves the ground state, then it goes undisturbed from the second cavity and leaves it empty. In both situations, the atom exits in the ground state, while the field in the two cavities is left in the following entangled state:

$$
\left|\Psi_{c}\right\rangle=\frac{1}{\sqrt{2}}\left(|0\rangle_{1}|1\rangle_{2}+|1\rangle_{1}|0\rangle_{2}\right)
$$

where the indices 1 and 2 refer to the first and the second cavity, respectively. The presence of one photon in any one of the cavities implies that the other is in the vacuum state, with a maximal quantum entanglement between the two possibilities. Once the atom is detected in the ground state, then we are ready for the next step of the teleportation.

The combined state of the atomic momentum $\left|\Psi_{a}\right\rangle$ and entangled field $\left|\Psi_{c}\right\rangle$ is given by

$$
\begin{aligned}
|\Psi\rangle= & \frac{c_{a}}{\sqrt{2}}\left(|0\rangle_{1}|1\rangle_{2}\left|+p_{0}\right\rangle+|1\rangle_{1}|0\rangle_{2}\left|+p_{0}\right\rangle\right) \\
& +\frac{c_{b}}{\sqrt{2}}\left(|0\rangle_{1}|1\rangle_{2}\left|-p_{0}\right\rangle+|1\rangle_{1}|0\rangle_{2}\left|-p_{0}\right\rangle\right) .
\end{aligned}
$$

The product can be extended in terms of the Bell basis

$$
\begin{aligned}
& \left|\Psi^{ \pm}\right\rangle=\frac{1}{\sqrt{2}}\left(\left|+p_{0}\right\rangle|0\rangle_{1} \pm\left|-p_{0}\right\rangle|1\rangle_{1}\right), \\
& \left|\Phi^{ \pm}\right\rangle=\frac{1}{\sqrt{2}}\left(\left|+p_{0}\right\rangle|1\rangle_{1} \pm\left|-p_{0}\right\rangle|0\rangle_{1}\right),
\end{aligned}
$$

and is given by the following:

$$
\begin{aligned}
|\Psi\rangle= & \frac{1}{2}\left[\left|\Psi^{+}\right\rangle\left(c_{a}|1\rangle_{2}+c_{b}|0\rangle_{2}\right)+\left|\Psi^{-}\right\rangle\left(c_{a}|1\rangle_{2}-c_{b}|0\rangle_{2}\right)\right. \\
& \left.+\left|\Phi^{+}\right\rangle\left(c_{a}|0\rangle_{2}+c_{b}|1\rangle_{2}\right)+\left|\Phi^{-}\right\rangle\left(c_{a}|0\rangle_{2}-c_{b}|1\rangle_{2}\right)\right] .
\end{aligned}
$$

The next step of teleportation is the measurement of the Bell states, i.e., a joint measurement of atomic momentum state and field inside the first cavity. It is clear from Eq. (6) that each of the Bell states is correlated with a certain superposition of zero- and one-photon field in the second cavity. Due to the measurement, the second cavity collapses into one of the four possibilities, each occurring equally with a probability of $\frac{1}{4}$, that carries the information of the atomic center-of-mass momentum state. In order to perform the measurement, first we need to disentangle the Bell state. This can be done by injecting slow two-level atoms initially in their ground state inside the first cavity. The atoms are in an unknown superposition of momentum states $\left|+p_{0}\right\rangle$ or $\left|-p_{0}\right\rangle$ along the direction of the wave propagation as defined by Eq. (1), representing the center-of-mass motion of the atom (see Fig. 1). The cavity field that is tuned to the atomic transition is in a superposition of zero and one photon. Atoms while passing through the cavity interact with the field in the Bragg regime and can absorb or emit a photon. In response, it may get deflected in the direction of wave propagation by an integral multiple of photon momentum $\hbar k$, where $k$ represents the propagation vector. If the atom experiences complete cycles of consecutive excitation and deexcitation, i.e., even number of interactions, then it leaves the cavity in the same state conserving energy. During the whole process, the total change in the momentum of the atom is $\pm l \hbar k$, where $l$ is an even integer. The conservation of momentum requires

$$
p_{\text {out }}=p_{\text {in }}+l \hbar k,
$$

where $p_{\text {in }}$ corresponds to the momentum of the incoming atom and $p_{\text {out }}$ is the momentum of the outgoing atom after $l$ interaction with the cavity field. Under the Bragg regime, there are only two possible directions of the atomic momentum that are symmetric with respect to the transverse directions, i.e., $y$ axis as shown in the Fig. 1. Along these directions, the magnitude of the atomic momentum on both sides remains the same. The initial magnitude of the atomic momentum is assumed to be $p_{i n}=l \hbar k / 2$, where $l$ is an even integer. The conservation of kinetic energy requires that

$$
\frac{p_{\text {out }}^{2}}{2 M}=\frac{\left|p_{\text {in }}+l \hbar k\right|^{2}}{2 M}
$$

where $M$ is the atomic mass. If $p_{0}$ denotes the initial and final magnitudes of the momentum of the atomic center of mass along the wave propagation direction inside the cavity, then Eq. (8) has following two solutions: $l=0$ and

$$
l=-l_{0}=-\frac{2 p_{0}}{\hbar k} .
$$

Here, the first solution corresponds to the incoming atom and the second to the scattering component of the atomic beam, conserving both energy and momentum. Under this condition, when the atom with initial momentum $p_{0}$ enters the cavity that is in a vacuum state, then it emerges with the same initial momentum. However, if cavity contains one photon, then it emerges in the $-p_{0}$ state as shown in the Fig. 1. The same result applies to the situation where we have the initial atom in momentum state $-p_{0}$.

The above idea is used to design quantum logic gates in Ref. [18], where the control bit is defined through the photon number in the cavity field which is either in logic " 0 " or " 1 ," whereas, the controlled bit is characterized by the welldefined momentum of the center-of-mass motion of the atom. The initial and final states of the cavity field and atomic center-of-mass momentum are summarized in Table 
TABLE I. Table of the cavity states and the atomic momentum state acting as a quantum controlled-NOT gate.

\begin{tabular}{ccc}
\hline \hline Initial states & & Final states \\
\hline$\left|0, p_{0}\right\rangle$ & $\rightarrow$ & $\left|0, p_{0}\right\rangle$ \\
$\left|0,-p_{0}\right\rangle$ & $\rightarrow$ & $\left|0,-p_{0}\right\rangle$ \\
$\left|1, p_{0}\right\rangle$ & $\rightarrow$ & $\left|1,-p_{0}\right\rangle$ \\
$\left|1,-p_{0}\right\rangle$ & $\rightarrow$ & $\left|1, p_{0}\right\rangle$ \\
\hline \hline
\end{tabular}

I, which shows the operation of quantum controlled-NOT logic gate. A detailed calculation to obtain the conditions that ensure the operation of quantum logic gates is presented in Ref. [18].

Applying the idea of quantum controlled-NOT logic gates, the Bell states defined by Eqs. (4) and (5) evolve in the following way:

$$
\left|\Psi^{ \pm}\right\rangle \rightarrow \frac{1}{\sqrt{2}}\left(|0\rangle_{1} \pm|1\rangle_{1}\right)\left|+p_{0}\right\rangle,
$$

and

$$
\left|\Phi^{ \pm}\right\rangle \rightarrow \frac{1}{\sqrt{2}}\left(|1\rangle_{1} \pm|0\rangle_{1}\right)\left|-p_{0}\right\rangle .
$$

Now the Bell states are disentangled and the atoms can be detected in the two directions that indicate whether the atom plus cavity system is in $\Psi^{ \pm}$or $\Phi^{ \pm}$state.

In order to completely determine the Bell state, next we propose a scheme that is based on cavity QED. We send a resonant two-level atom initially in the first ground state through the first cavity which is a superposition of zero- and one- photon states. If the atom encounters a zero photon, then it goes undisturbed, otherwise it may absorb one photon from the cavity and get excited. Halfway inside the cavity, we apply a strong $\pi / 2$ pulse that brings the atom in a superposition of the excited and ground states. It may be pointed out that this region should be small enough so that the atom does not undergo any transition. Now we have two possibilities: (i) if the atom is in the ground state, then it may go undisturbed through the cavity and exit in the ground state leaving the zero-photon state inside the cavity, (ii) if the atom is in the excited state, then it may contribute a photon inside the cavity and exit in the ground state. In both situations, the atom leaves the cavity in the ground state carrying no information, whereas the cavity field will be left either in zero- or one-photon state. This leads to the evolution of joint states [see Eqs. (10) and (11)] in the following way:

$$
\begin{aligned}
\frac{1}{\sqrt{2}}\left(|0\rangle_{1} \pm|1\rangle_{1}\right)|b\rangle & \rightarrow \frac{1}{\sqrt{2}}\left(|0\rangle_{1}|b\rangle \pm|0\rangle_{1}|a\rangle\right) \\
& \rightarrow \frac{1}{\sqrt{2}}\left(|0\rangle_{1}(|a\rangle+|b\rangle) \pm|0\rangle_{1}(|a\rangle-|b\rangle)\right. \\
& \rightarrow\left\{\begin{array}{l}
|1\rangle_{1}|b\rangle \\
|0\rangle_{1}|b\rangle
\end{array}\right.
\end{aligned}
$$

We, therefore, obtain

$$
\left|\Psi^{ \pm}\right\rangle \rightarrow \frac{1}{\sqrt{2}}\left(|1\rangle_{1} \pm|0\rangle_{1}\right)\left|+p_{0}\right\rangle|b\rangle \rightarrow\left\{\begin{array}{l}
\left|+p_{0}\right\rangle|1\rangle|b\rangle \\
\left|+P_{0}\right\rangle|0\rangle|b\rangle
\end{array}\right.
$$

similarly,

$$
\left|\Phi^{ \pm}\right\rangle \rightarrow \frac{1}{\sqrt{2}}\left(|1\rangle_{1} \pm|0\rangle_{1}\right)\left|-p_{0}\right\rangle|b\rangle \rightarrow\left\{\begin{array}{l}
\left|-p_{0}\right\rangle|1\rangle|b\rangle \\
\left|-p_{0}\right\rangle|0\rangle|b\rangle
\end{array}\right.
$$

The joint measurement can be achieved by detecting the final momentum state $\pm\left|p_{0}\right\rangle$ and the field inside the first cavity which is either in zero- or one-photon state.

The final momentum states after the Bragg scattering of atoms with the standing-wave field in the first cavity can be measured by measuring the far-field position distribution. In the far field of the interaction region, the atomic position distribution can be obtained by exciting the atoms with a resonant laser beam and then detecting the fluorescence photons. The measured position distribution yields information about the atomic momentum state [19]. To distinguish between zero- and one-photon states, a two-level atom initially in the ground state and resonant with the cavity field can be injected inside the cavity. If the atom encounters zero photon, then it leaves the cavity in the ground state, otherwise, it will absorb one photon from the cavity field and exit in the excited state. Thus, a detection of the atom in its excited or ground state will determine whether the cavity field is in zero- or one-photon state. The outcome of the measurements can be transmitted to the receiver through a classical channel. Finally, a receiver can apply an appropriate unitary transformation to the field inside the second cavity [which is projected in one of the four possibilities appearing in Eq. (6)], and reconstruct the momentum state of the atom with $|0\rangle_{2}$ and $|1\rangle_{2}$ replaced by $\left|+p_{0}\right\rangle_{2}$ and $\left|-p_{0}\right\rangle_{2}$.

Here we discuss the unitary transformation that a receiver must apply to the field inside the second cavity to complete the teleportation procedure. If the state of the second cavity is $c_{a}|0\rangle+c_{b}|1\rangle$, then it is the original state and no operation is required. For $c_{a}|0\rangle-c_{b}|1\rangle$, we need to change the phase of state $|1\rangle$. This can be achieved by passing an atom initially prepared in a superposition of state $(|a\rangle+|b\rangle) / \sqrt{2}$ and choosing the interaction time such that ground state acquires a phase of -1 . If the atom is detected in state $|b\rangle$, then the cavity field reduces to $c_{a}|0\rangle+c_{b}|1\rangle$ which is the original state. However, if the atom is detected in excited state $|a\rangle$, then we need to repeat the process again, until the atom is detected in state $|b\rangle$. Now if the second cavity is in state $c_{b}|0\rangle+c_{a}|1\rangle$, then we need to swap the probabilities. This can be achieved by passing an atom initially prepared in the ground state and choosing the interaction time such that the field coherence is transferred to the atom leading to the state $\left(c_{a}|a\rangle+c_{b}|b\rangle\right)$. In the next step, we apply a strong classical field such that the states of the atom switch to $\left(c_{b}|a\rangle\right.$ $\left.+c_{a}|b\rangle\right)$. Atom is then passed through an empty cavity, which at the exit leaves the cavity in the original state 
$\left(c_{a}|0\rangle+\left(c_{b}|1\rangle\right)\right.$. Here we need an extra cavity, however, in principle, it is not really necessary and the transformation can be obtained in a single cavity by manipulating the field time dependently. For the state $c_{a}|1\rangle-c_{b}|0\rangle$ ), we need both swapping of the probabilities and a phase shift, which can be obtained as discussed in the last two cases.

In summary, we propose a simple scheme for teleportating an unknown superposition of an atomic center-of-mass momentum state from sender's end to a superposition of the cavity field at receiver's end. The scheme relies on the ideas from atom optics and cavity QED. The measurement of Bell states are realized by employing a quantum controlled-NOT gate using atomic scattering by a standing-wave field in a Bragg regime and then using cavity QED. It may be pointed out that throughout the paper, we neglect atomic spontaneous emission and cavity losses. Recent developments on the realization of the Bragg diffraction of atoms from the cavity field [20] and the possibility of generating photon number states inside a high- $Q$ cavity [21] makes the proposed scheme experimentally realizable.

We would like to thank Tomas Opatrny for useful discussions. We gratefully acknowledge the support from the Air Force Research Laboratories (Rome, New York), DARPAQuIST, TAMU Telecommunications and Informatic Task Force (TITF) Initiative, and the Office of Naval Research. We would also like to thank RGC, Government of Hong Kong, for a research grant. One of us (S.Q.) would like to thank the Ministry of Science \& Technology and Government of Pakistan for financial support.
[1] A. Einstein, B. Podolsky, and N. Rosen, Phys. Rev. 47, 777 (1935).

[2] J.S. Bell, Physics (Long Island City, N.Y.) 1, 195 (1964).

[3] C.H. Bennett, G. Brassard, C. Crepeau, R. Jozsa, A. Peres, and W.K. Wootters, Phys. Rev. Lett. 70, 1895 (1993).

[4] L. Davidovich, N. Zagury, M. Brune, J.M. Raimond, and S. Haroche, Phys. Rev. A 50, R895 (1994).

[5] J.I. Cirac and A.S. Parkins, Phys. Rev. A 50, R4441 (1994); H. Wienfurter, Europhys. Lett. 25, 559 (1994); M.H.Y. Moussa, Phys. Rev. A 55, R3287 (1997); S.-B. Zheng and G.-C. Guo, Phys. Lett. A 232, 171 (1997); C.S. Maierle, D.A. Lidar, and R.A. Harris, Phys. Rev. Lett. 81, 5928 (1998); S. Stenholm and P.J. Bardroff, Phys. Rev. A 58, 4373 (1998); L. Vaidman and N. Yoran, ibid. 59, 116 (1999); N. Linden and S. Popescu, ibid. 59, 137 (1999); M. Koniorczyk, J. Janszky, and Z. Kis, Phys. Lett. A 256, 334 (1999); S. Bose, P.L. Knight, M.B. Plenio, and V. Vedral, Phys. Rev. Lett. 83, 5158 (1999).

[6] M. Ikram, S.-Y Zhu, and M.S. Zubairy, Phys. Rev. A 62, 022307 (2000).

[7] M.S. Zubairy, Phys. Rev. A 58, 4368 (1998).

[8] L. Vaidman, Phys. Rev. A 49, 1473 (1994).

[9] S.L. Braunstein and H.J. Kimble, Phys. Rev. Lett. 80, 869 (1998).

[10] T.C. Ralph and P.K. Lam, Phys. Rev. Lett. 81, 5668 (1998); G.J. Milburn and S.L. Braunstein, Phys. Rev. A 60, 937 (1999).

[11] D. Bouwmeester, J.-W. Pan, K. Mattle, M. Eibl, H. Weinfurter, and A. Zeilinger, Nature (London) 390, 575 (1997).
[12] D. Boschi, S. Branca, F. DeMartini, L. Hardy, and S. Popescu, Phys. Rev. Lett. 80, 1121 (1998).

[13] A. Furusawa, J.L. Sorensen, S.L. Braunstein, C.A. Fuchs, H.J. Kimble, and E.S. Polzik, Science 282, 706 (1998).

[14] E. Lombardi, F. Sciarrino, S. Popescu, and F. De Martini, Phys. Rev. Lett. 88, 070402 (2002); S. Giacomini, F. Sciarrino, E. Lombardi, and F. De Martini, Phys. Rev. A 66, 030302(R) (2002).

[15] A. Kuzmich and E.S. Polzik, Phys. Rev. Lett. 85, 5639 (2000).

[16] T. Opatrny and G. Kurizki, Phys. Rev. Lett. 86, 3180 (2001).

[17] A.S. Parkins and H.J. Kimble, e-print quant-ph/9909021.

[18] A.A. Khan and M.S. Zubairy, Fortschr. Phys. 46, 417 (1998).

[19] S. Dürr and G. Rempe, Phys. Rev. A 59, 1495 (1999).

[20] P.E. Moskowitz, P.L. Gould, S.R. Atlas, and D.E. Pritchard, Phys. Rev. Lett. 51, 370 (1983); P.L. Gould, G.A. Ruff, and D.E. Pritchard, ibid. 56, 827 (1986); P.J. Martin, P.L. Gould, B.G. Oldaker, A.H. Miklich, and D.E. Pritchard, Phys. Rev. A 36, 2495 (1987); P.J. Martin, B.G. Oldaker, A.H. Miklich, and D.E. Pritchard, Phys. Rev. Lett. 60, 515 (1988); P.L. Gould, P.J. Martin, G.A. Ruff, R.E. Stoner, J.L. Picque, and D.E. Pritchard, Phys. Rev. A 43, 585 (1991); L.S. Goldner, C. Gerz, R.J.C. Spreeuw, S.L. Rolston, C.I. Westbrook, W.D. Phillips, P. Marte, and P. Zoller, Phys. Rev. Lett. 72, 997 (1994); D.M. Giltner, R.W. McGowan, and S.A. Lee, ibid. 75, 2638 (1995); D.M. Giltner, R.W. McGowan, and S.A. Lee, Phys. Rev. A 52, 3966 (1995); S. Kunze, S. Dürr, and G. Rempe, Europhys. Lett. 34, 343 (1996).

[21] S. Brattke, B.T.H. Varcoe, and H. Walther, Phys. Rev. Lett. 86, 3534 (2001). 